\title{
Hydrophobic Biomimetic Nanoparticles drives Size-dependent Remodelling in Asymmetric Bilayers
}

\author{
Sang Young Noh, ${ }^{*,+,}$ and Rebecca Notman ${ }^{*,+, \neq}$ \\ $\dagger$ Center for Scientific Computing, University of Warwick, Coventry, Warwickshire, UK \\ $\ddagger$ Department of Chemistry, University of Warwick, Coventry, Warwickshire, UK \\ E-mail: S.Y.Noh@Warwick.ac.uk; R.Notman@Warwick.ac.uk
}

\begin{abstract}
The interactions between heterogeneous components in a biomimetic bilayer can control its physical properties such as its rigidity, local and bulk curvature and propensity towards phenomena such as membrane fission and fusion. In particular, nanoparticles (NPs) have been subjects of intense interest due to their similar scale to the bilayer width and its ability to affect local membrane structure. Generally, it is understood that hydrophobic components are energetically favoured to adsorb within the hydrophobic interior of a biomimetic bilayer. However, how such NPs interact in the presence of heterogeneous aggregates in the bilayer has been the subject of much debate. To better understand the effects of the integration of nanoscale components on heterogeneous mixed bilayer, we have simulated a series of generic hydrophobic NPs interacting with a phase-separating two-component surfactant bilayer. We find that the hydrophobic NP tends to aggregate at the phase interface, acting as a line tension relaxant i.e. a lineactant on the phase separated interface, which results in a variety of demixing behavior. We demonstrate that depending on the size of the NP, the localized softening
\end{abstract}


of surfactants and the formation of a mixing gradient of surfactants can drive the a cap/bud formation around the NP, as well as the formation of a NP-micelle structure.

\section{Introduction}

Lipids and similar polyether/polyethylene type molecules form the building blocks of a whole range of materials ranging from traditional manufacturing ingredients such as detergents and

paints, ${ }^{1}$ to more recent, complex, applications such as in bio-sensing devices, drug delivery capsules and biomedical apparatus. ${ }^{2-6}$ Whether the lipid/polymer is biological or artificial, its basic building blocks comprises of short hydrophilic headgroups and longer hydrophobic tails. Attaching these different groups into a single lipid type species, allows the aggregation of these molecules - the hydrophilic headgroups can associate with the surrounding bulk water surrounding it, and the water-avoiding hydrophobic tailgroups aggregate away from the headgroup and water. Depending on the structure and the molecular species which make up the lipid/polymer moiety, the bulk structure can form a large array of structures ranging from a monolayer micelle to a semi-flexible planar bilayer or a bilayer vesicle. Bilayers also constitute the semi-permeable vesicles that build the major organelles within biological organisms. The preferential interactions between the molecular components, due to steric preference or direct intermolecular energetic preference has been hypothesized to drive numerous complex processes within the bilayer.

The pioneering work by Simons and Ikonen ${ }^{7}$ illustrated the possible existence of patches or rafts with heterogeneous bilayers - structures ranging from 10-200 nm radius - enriched semi-circular domains that are stabilized by heterogeneous components, forming what are known as liquid-ordered $\left(l_{o}\right)$ phases (which primarily make up the patch formation) from the liquid-disordered $\left(l_{d}\right)$ components. While the definitive evidence for the existence of rafts remains to be seen, the study does illustrate the need for study in how the heterogeneous components can influence the structure of the bilayer. The formation of patches 
in a mixed bilayer has been suggested to form the platform for complex macro-structures, such as membrane proteins to control the translocation of particles and ions across the bilayer. ${ }^{8}$ In general, it is thought that the energetic cost of the raft formation depends on two main factors - the free energy gain when like lipid species coagulate together (either through packing energy or hydrophobic interaction match), and secondly, minimization of the free energy cost of the domain boundaries. It has been suggested that the hybrid lipid species (with a saturated and unsaturated tail), such as monosaturated POPC may control the type and size of the domains formed, through a controlled 'loosening' of the membrane domain line tension. Other stabilizing factors include the presence of cholesterol (CHOL), which aggregates to the saturated tailgroups, therefore acting as a stabilizing element to $l_{o}$ phases. ${ }^{9}$

In addition to experimental evidence, molecular simulation data have also demonstrated the importance of heterogeneous components upon the phase change within mixed bilayer environments. Hakoyoban et $a l^{10}$ for example, used coarse-grained molecular dynamics (CGMD) with the MARTINI ${ }^{11}$ force field to systematically study a ternary DOPC/DLPC/CHOL system, with added variations in the headgroup and tailgroup regions. The study suggested that the rigidity of lipid/cholesterol species has a significant effect on stabilizing/slowing the phase separation between $l_{o}$ and and $l_{d}$ species in a heterogeneous lipid bilayer system, which closely follows experimental observations. In addition, the interaction of raft-like aggregates with membrane proteins seems to suggest that these can modulate the rate of phase separation. For example, Fowler et al ${ }^{12}$ demonstrated through molecular simulation that the distribution and density of peripheral membrane proteins can change the bending rigidity. Hence, the domain formation in unsaturated/saturated mixtures have shown to be slowed by the presence of such 'linking' proteins, where it can reduce the unfavourable contact in the interface between the $l_{o}$ and $l_{d}$ phases. ${ }^{12}$ In addition, hybrid lipid structures (lipids with both saturated and unsaturated tails) has been shown to act in a similar way. For example, Rosetti et $a l^{13}$ demonstrated through quarternary mixtures of PAPC/DAPC/DPPC/CHOL 
mixtures that hybrid lipid type structures reduce the hydrophobic mismatch area between the $l_{o}$ and $l_{d}$ regions. Furthermore, all-atomic (AA) simulation results by Hassan-Zadeh et $a l^{14}$ demonstrated that POPC/PLPC/PAPC type lipids essentially 'even-out' the physical characteristics between the $l_{o}$ and $l_{d}$ phase, which effectively reduced the domain line tension. Hence, the primary role of line tension relaxants (otherwise referred to as lineactants) is hypothesized to be modulating the rate of phase separation by reducing the contact surface between the unfavourable components.

Due to their potential for applications such as targeted drug-delivery, and their high biocompatibility, nanoparticles (NPs), nanometer-scale objects of various radius and anisotropy, have recently been a subject of intense interest in relation to their application with bilayer systems. As indicated from past studies, the primary driving force for the phase separation into $l_{o} / l_{d}$ phases is the line tension between the phases. NPs with hydrophobic characteristics are interesting to consider as it can adsorb within the interior of the bilayer surface. Therefore, understanding how it may influence the arrangement of components within the bilayer is crucial to understanding and designing better nanoscale applications. For example, Barnoud et $a l^{15}$ observed that domain stability depends on the presence of aliphatic species/NPs (Octane, Hexadecane, Cyclohexane) near the $l_{d} / l_{o}$ domains. In addition, through mesoscopic simulations, Cheung ${ }^{16}$ also demonstrated the control of raft-like domains with mesoscaledynamics in the presence of hydrophobic NPs, suggesting that a hydrophobic NP and a hydrophobic protein analogue can nucleate domains and act as lineactants, in a similar manner to membrane proteins.

While it is clear that the interactions of NPs can affect the phase separating behaviour of mixed bilayers, to the authors best knowledge, the systematic study of size-dependent effects of hydrophobic NPs upon the rate and type of phase separation has not been studied before. In our work, we simulate a mixed bilayer with a hydrophobic NP of a series of increasing sized radius. We designed a continuum model of a hydrophobic NP to model the effects of a nanoscale hydrophobic component in a deliberately phase-separated biomimetic surface, 
which acts as a model for a mixed bilayer system with raft-like structures. We demonstrate that hydrophobic NPs of all sizes within this study locates itself within the interfaces between the phases, and may reflect the general behaviour of hydrophobic proteins and NPs within heterogeneous bilayers. In addition, we show that with larger radius, the NP drives a budding process that may lead to the formation of caps to a full-fledged budding.

\section{Methods}

\section{Model of the Bilayer}

We used the Shinoda-DeVane-Klein (SDK) CG-MD model ${ }^{17-19}$ (more recently updated to $\left.\mathrm{SPICA}^{20}\right)$. The polyethylene glycol $\left(\mathrm{C}_{12} \mathrm{E}_{2}\right)$ model used by Shinoda et al ${ }^{17,18}$ was the basis of the model used in this work. The model has been parameterized against thermodynamic properties (densities, interfacial tensions, transfer free energies) and has been applied successfully by itself or as a model to study a range of soft matter systems. ${ }^{20-25}$ A brief description of their coarse-graining approach follows here - within this model 3-5 heavy atoms are represented by a single interaction site or "bead" (one water bead represents three water molecules). The surfactant molecule was described using four $\mathrm{CG}$ bead types: $\mathrm{OA}\left(-\mathrm{CH}_{2} \mathrm{OH}\right)$ and EO $\left(-\mathrm{CH}_{2} \mathrm{OCH}_{2^{-}}\right)$which represent the hydrophilic head group and $\mathrm{CM}\left(-\mathrm{CH}_{2} \mathrm{CH}_{2} \mathrm{CH}_{2^{-}}\right.$ ) and $\mathrm{CT} 2\left(\mathrm{CH}_{3} \mathrm{CH}_{2} \mathrm{CH}_{2^{-}}\right)$, which represent the hydrophobic tail groups. To produce a mixed bilayer, half of the $\mathrm{C}_{12} \mathrm{E}_{2}$ molecules were changed into modified equivalents, which has identical molecular mechanical properties. This initial configuration was produced by a randomized change of indices for $50 \%$ of the atoms of the bilayer data file. The modified lipid was produced by changing the Lennard-Jones (LJ) $\epsilon$ values for the non-bonded potentials between surfactant and mimic beads - the LJ parameters were changed so that we could produce system which show a self-preferential phase separation to take into account the large variety of phase separation mechanisms which can be seen in real lipid bilayers. The tabulated values for these systems are shown in the supplementary information. The 
schematic of the AA to CG mapping, as well as the modification of the surfactant is shown in Figure 1(a). The non-bonding potentials between the $\mathrm{C}_{12} \mathrm{E}_{2}$ and $\mathrm{C}_{12} \mathrm{E}_{2}-\mathrm{M}$ has been modified to show a greater extent of intermolecular repulsion between the original and mimic beads. Figure 1(b) and (c) shows the illustration of the NPs used for this study and the top-down view of the $\mathrm{C}_{12} \mathrm{E}_{2} / \mathrm{C}_{12} \mathrm{E}_{2}-\mathrm{M}$ bilayer system used respectively.

\section{Model of the NPs}

The NPs were treated as a collection of uniformly distributed interaction sites i.e. the particle was treated as a surface-to-molecule potential, based upon Hamaker's model for modeling the non-bonding potential of surfaces. ${ }^{26,27}$ To represent the hydrophobic NPs, the interaction sites of the particle took the LJ parameters of the bead type CT2; the interactions between the NP and CG beads were derived from integrating the interaction potential between a CG bead and an interaction site within the NP over the particle's volume. Assuming that the interaction between a CG bead and single interaction site can be described through the modified LJ functions (Equations 1 and 2) the interaction between a NP and a CG bead may be written as

$$
\begin{gathered}
U_{N P}^{12-4}(r, R)=\frac{2 \sqrt{3} \pi \rho \epsilon \sigma^{12} R^{3}}{15} \frac{5 R^{6}+45 R^{4} r^{2}+63 R^{2} r^{4}+15 r^{6}}{\left(r^{2}-R^{2}\right)^{9}} \\
-\frac{3 \pi \rho \epsilon \sigma^{4} R}{r^{2}-R^{2}}+\frac{3 \sqrt{3} \pi \rho \epsilon \sigma^{4}}{2 r} \ln \left[\frac{r-R}{r+R}\right] \\
U_{N P}^{9-6}(r, R)=9 \pi \rho \epsilon \sigma^{9} R^{3} \frac{3 R^{4}+42 R^{2} r^{4}+35 r^{4}}{35 r\left(r^{2}-R^{2}\right)^{6}}-\frac{9 \pi \rho \epsilon \sigma^{6} R^{3}}{r^{2}-R^{2}}
\end{gathered}
$$

where $r$ is the distance from the NP center, $\rho$ is the density of the NP and $R$ is the NP radius. The density of the NP was taken to be $1000 \mathrm{~kg} \mathrm{~m}^{-3}$ (based on the density of water). Full derivations of these formulae are given in the supplementary information. The parameters

$\epsilon$ and $\sigma$ are the Van der Waals parameters for the W (hydrophilic) or CM (hydrophobic) CG beads. The interaction potential between the NP and solvent bead is taken as a simple 
interpolation of the hydrophilic and hydrophobic potentials (Equation 3):

$$
U_{N P}^{\text {type }}(r)=(x) U_{N P}^{\text {hydrophilic }}(r)+(1-x) U_{N P}^{\text {hydrophobic }}
$$

where we set $x=0$ to ensure a fully hydrophobic NP for each size. Plots of the interaction potentials for each NP are provided in supplementary information (Figure S4). Three $R$ values were used for creating the NPs - $R=1.0,1.5$ and $2.0 \mathrm{~nm}$. The $1.0 \mathrm{~nm}$ radius NP represents a NP that is comparable to the tailgroup (CM and CT2) length of the surfactant, while the $2.0 \mathrm{~nm}$ radius NP represents a NP with a width that is comparable to the thickness of the bilayer, the $1.5 \mathrm{~nm}$ radius being the intermediate. For each $\mathrm{R}$, the hydrophobic interaction between the $\mathrm{NP}$ and $\mathrm{C}_{12} \mathrm{E}_{2} / \mathrm{C}_{12} \mathrm{E}_{2}-\mathrm{M}$ is identical.

\section{Simulation Parameters}

All simulations were performed using the LAMMPS molecular dynamics package. ${ }^{28}$ To generate the initial configuration of the bilayer, $3500 \mathrm{CG}$-beads of the $\mathrm{C}_{12} \mathrm{E}_{2} / \mathrm{C}_{12} \mathrm{E}_{2}-\mathrm{M}(500$ molecules in total) were used to arrange a bilayer in a simulation box ranging from dimensions $80 \AA \times 80 \AA \times 200 \AA$ with the rest of the box being filled with 14328 water beads. This system was equilibrated using a $N V T$ ensemble for $1 \mathrm{~ns}$ simulation time with increasing timesteps to ensure the overlaps between molecules were resolved and to ensure the system did not fluctuate substantially. To reduce the likelihood of finite size effects, the simulation box was replicated once in each of the $x$ and $y$ directions, meaning that the simulation box was expanded from $80 \AA \times 80 \AA \times 200 \AA$ to $160 \AA \times 160 \AA \times 200 \AA .14000 \mathrm{CG}$ beads of the $\mathrm{C}_{12} \mathrm{E}_{2} / \mathrm{C}_{12} \mathrm{E}_{2}$-M was used to construct the larger bilayer, which equals to 2000 molecules. This new system was also subjected to 1 ns of $N V T$ equilibration in the same manner as the smaller bilayer. To insert the NP, the water beads were removed, the simulation box dimension in the direction normal to the bilayer (z-direction) was set to $200 \AA$ and the NP was inserted into the system $100 \AA$ above the center of mass of the bilayer followed by 
re-solvation. For productions runs, the system was simulated in the NPT-ensemble, with temperature and pressure set to $303 \mathrm{~K}$ and 1 atm respectively. The temperature and pressure were controlled using a Nosé-Hoover thermostat and barostat, ${ }^{29,30}$ both with relaxation times of 0.2 ps. The equations of motion were integrated using the rRESPA multiple-timestep algorithm ${ }^{31}$ with 2.0 fs inner (bonded) and 10.0 fs (non-bonded) outer timesteps. Non-bonded interactions were truncated at $r_{\text {cut }}=15 \AA$. Periodic Boundary Conditions (PBCs) were set in the $x$ and $y$ directions. A test run of 200 ns was run to ensure that the surfactant bilayer had reached consistency in it's properties with past experimental and simulation results. This was followed by the production run over $1000 \mathrm{~ns}$ in the NPT ensemble. Two series of simulations were run, where the unrestrained simulations kept the lipids and the NPs under no external restraints (representing the true free trajectory simulation), while the restrained simulations applied an Umbrella potential at the bilayer center to ensure the effect of any potential budding would be restrained (restrained in the bilayer normal plane, but free in the bilayer lateral plane), with the purpose of analyzing the NPs locational preference around the mixed bilayer (representing a artificial trajectory to study the phase behaviour of the surfactants around the NP).

\section{Membrane Domain and Budding Energetics}

We have approached the problem of membrane energetics in the way as described by Wolff et $a l^{32,33}$ and Lipowsky, ${ }^{34,35}$ which involves dividing up the bending, interface and composition energetics, following on from the elastic properties of a bilayer described by Helfrich. ${ }^{36}$ The approach entails dividing up the bilayer conformation around the domain and the NP in terms of the bending energy (the cost of increasing the curvature around the NP), the edge energy (the unfavourable energetic contribution between the domains) and the adhesion energy (the energetic cost of adhering on the NP surface). The bending energy term is defined by:

$$
E_{\text {bending }}=2 \pi L^{2} \kappa\left[\left(C-C_{0}\right)^{2}+\left(C+C_{0}\right)^{2}\right]
$$



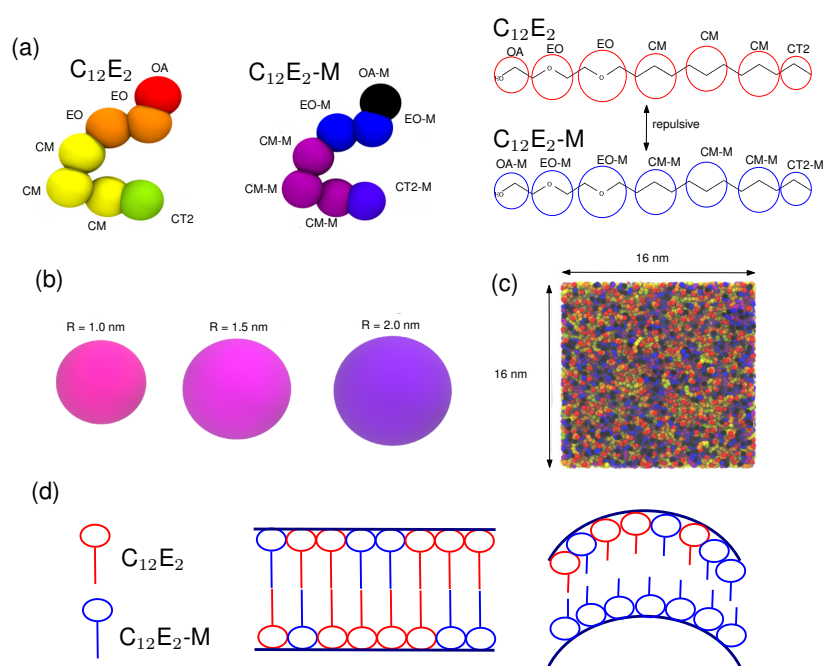

$\phi_{1}=\phi_{2}$

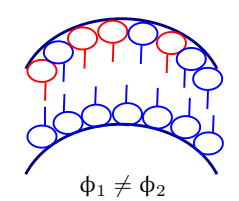

Figure 1: (a) shows the schematic and $\mathrm{CG}$ mapping of the $\mathrm{C}_{12} \mathrm{E}_{2}$ and the $\mathrm{C}_{12} \mathrm{E}_{2}-\mathrm{M}$ surfactants, and (b) shows the schematics and sizes of the hydrophobic NPs. (c) shows the top-down starting configuration and dimensions of the surfactant bilayer. (d) shows the scenario where the top and bottom monolayer are well-mixed, which is equivalent to the top (1) and bottom (2) monolayer composition being equal $\left(\phi_{1}=\phi_{2}\right)$, and the case where the one monolayer is significantly more domain-like $\left(\phi_{1} \neq \phi_{2}\right)$.

Where $\kappa$ is the bending rigidity (taken as $5.4 \mathrm{k}_{B} \mathrm{~T}$ for $\mathrm{C}_{12} \mathrm{E}_{2}$, as computed by Kurtisovski et $\left.a l^{37}\right), L$ is the radius of the dimple/cap, $\mathrm{C}$ is the curvature of the dimple/cap, and $\mathrm{C}_{0}$ the spontaneous curvature of the bilayer. Here, we can estimate the curvature as $\frac{1}{R}$, if we assume that the center of the NP position is aligned with the peak of the cap/micelle. The energetic cost of forming the edge also needs to be taken into account - the domain edge energy can be defined as:

$$
E_{\text {edge }}=2 \pi L \gamma \sqrt{1-(L C / 2)^{2}}
$$

where $\gamma$ indicates the line tension. To measure $\gamma$, it is required to measure the unfavourable interaction forces at the phase interface - originating from the difference in intermolecular potentials and/or structural variations. To infer $\gamma$, we used a stabilized phase-separated system which had stabilized domain boundaries, and extracted the stress tensor components for each $\mathrm{CG}$ bead of the $\mathrm{C}_{12} \mathrm{E}_{2}$ and $\mathrm{C}_{12} \mathrm{E}_{2}-\mathrm{M}$ components and averaged them over the intraplanar axes i.e. the $x$ and $y$ axes. This is extracted from the stress tensor components from the molecules in the system. The dependence of the budding on the phase separation 
of the bilayer can be described using the Landau expansion of the free energy of the $\phi$ is emphasized using the free energy powers of $\phi$ :

$$
E_{\text {phase }}=\pi L^{2} \frac{U}{\iota^{2}}\left[\frac{1}{2} \phi^{2}+\frac{1}{4} \phi^{4}\right]
$$

Where $U$ is the parameter that sets the energetic scale, which is defined as $2 \kappa$ as defined by Wolff, ${ }^{33}$ and $\iota$ represents $\frac{\kappa}{\gamma}$, the invagination length. As the simulations were carried out at a constant temperature ensemble, the reduced temperature term was taken as 1 to minimize the contribution of the change in temperature. Wolff et $a l^{32}$ estimated the 'incompatibility' energy (or the measure of the tendency of each bilayer component to disaggregate) from the Landau expansion of the monolayer composition, which is highly dependent on the monolayer compositions of the bilayer, which in turn affects the magnitude of the spontaneous curvature. Figure 1(d) shows an illustration of the possible scenarios of spontaneous curvature that may occur when the surfactant components mix. A flat bilayer domain may become spontaneously curved whenever an excess of a single lipid component is highly concentrated in a patch of the monolayer. Following the convention followed by Wolff, we have computed the monolayer compositions $\phi_{1}$ and $\phi_{2}$ (indices 1 and 2 representing each monolayer) around the NP in a $8 \mathrm{~nm}$ radius around the NP, to modify the composition dependent spontaneous curvature:

$$
\begin{aligned}
& \phi_{1}=\phi_{1}^{\mathrm{C}_{12} \mathrm{E}_{2}}-\phi_{1}^{\mathrm{C}_{12} \mathrm{E}_{2}-\mathrm{M}} \\
& \phi_{2}=\phi_{2}^{\mathrm{C}_{12} \mathrm{E}_{2}}-\phi_{2}^{\mathrm{C}_{12} \mathrm{E}_{2}-\mathrm{M}}
\end{aligned}
$$

Hence, we can compute the average composition of both monolayers as:

$$
\phi_{\mathrm{av}}=\frac{\phi_{1}+\phi_{2}}{2}
$$

The composition difference was averaged for every $1 \AA$ intervals away from the NP center. Each of the monolayer compositions around the NP region was analyzed to measure how 
the interaction of the surfactants of the bilayer affected the local compositions around the NP. Hence, by analyzing this composition data, we can estimate the extent of the monolayer incompatibility near the NP radius. This composition profile was averaged over every $200 \mathrm{~ns}$ intervals over the unrestrained simulations to analyze the evolution of the composition over the course of the simulation. The adsorption/adhesion energy $E_{\text {adhesion }}$ of the surfactants to the NP surface is another key factor. A negative $\mathrm{E}_{\text {adhesion }}$ is responsible

for the formation of the micelle/bud, while $\mathrm{E}_{\text {bending }}, \mathrm{E}_{\text {edge }}$ and $\mathrm{E}_{\text {phase }}$ are the equivalent to the energetic cost required. To compute $\mathrm{E}_{\text {adhesion }}$, we utilized the group/group command (https://lammps.sandia.gov/doc/compute_group_group.html) to compute the total energy of interaction between the NPs and the $\mathrm{C}_{12} \mathrm{E}_{2} / \mathrm{C}_{12} \mathrm{E}_{2}-\mathrm{M}$ groups. We can consider the sum of the $\mathrm{E}_{\text {bending }}, \mathrm{E}_{\mathrm{phase}}$, and $\mathrm{E}_{\text {edge }}$ as the factors that compose the energetic barrier to dim$\mathrm{ple} /$ vesicle formation, and therefore can be combined to form the $\mathrm{E}_{\text {total }}$ term:

$$
E_{\text {total }}=E_{\text {bending }}+E_{\text {edge }}+E_{\text {phase }}
$$

\section{Tail Order Parameter}

The averaged nematic tail order parameter around the NP computed. The tail order parameter is defined as:

$$
S=\frac{3}{2}\left\langle\cos ^{2} \theta\right\rangle-1
$$

where $\theta$ is the angle between the bilayer normal and the chain segment of the surfactant. The averaged values were computed in $2 \AA$ intervals away from the surface of the NPs to observe the change in the surfactant arrangement as a function of time. 
Table 1: Simulation parameters for each mixed bilayer/NP simulation.

\begin{tabular}{|c|c|c|c|c|}
\hline NP Radius (nm) & $\mathbf{C}_{12} \mathbf{E}_{2}(\mathbf{N})$ & $\mathbf{C}_{12} \mathbf{E}_{2}-\mathbf{M}(\mathbf{N})$ & $t(\mathbf{n s})$ & Force $\left(\mathrm{kcal} \mathrm{mol}^{-1} \AA^{-2}\right)$ \\
\hline- & 1000 & 1000 & 1000 & - \\
\hline 1.0 & 1000 & 1000 & 1000 & - \\
\hline 1.5 & 1000 & 1000 & 1000 & - \\
\hline 2.0 & 1000 & 1000 & 1000 & 25 \\
\hline 1.0 & 1000 & 1000 & 1000 & 25 \\
\hline 1.5 & 1000 & 1000 & 1000 & 25 \\
\hline 2.0 & 1000 & 1000 & 1000 & - \\
\hline
\end{tabular}

Table 2: Energetic parameters values used for curvature calculations.

\begin{tabular}{|c|c|c|c|c|c|c|}
\hline $\mathbf{N P}$ size $(\mathbf{n m})$ & $\mathbf{C}\left(\mathbf{n m}^{-1}\right)$ & $\gamma(\mathbf{N})$ & $\left.\frac{\kappa}{\sigma} \mathbf{n m}\right)$ & $\left.\mathbf{A}_{\mathbf{N P}_{\text {cap }}} \mathbf{( n m}^{2}\right)$ & $\mathbf{L} \mathbf{( n m})$ & $\mathbf{L C}$ \\
\hline 1.0 & 0.357 & $2.9607 \times 10^{-12}$ & 22.59 & $11.32 \pi$ & 2.8 & 0.9996 \\
\hline 1.5 & 0.303 & $2.9607 \times 10^{-12}$ & 22.59 & $15.79 \pi$ & 3.3 & 0.9990 \\
\hline 2.0 & 0.2 & $2.9607 \times 10^{-12}$ & 22.59 & $20.85 \pi$ & 3.8 & 0.7600 \\
\hline
\end{tabular}

\section{Results and Discussion}

\section{Restrained and Unrestrained Mixed Bilayer Systems with/without the NPs}

Table 1 shows the tabulated simulations involved in this work, where the top 4 simulations shows the free trajectories, while the bottom 3 shows the restrained NP simulations. Figure 2 (a) shows the snapshots of the $\mathrm{C}_{12} \mathrm{E}_{2} / \mathrm{C}_{12} \mathrm{E}_{2}-\mathrm{M}$ with the $R=1.0,1.5$ and $2.0 \mathrm{~nm}$ radius simulations. The mixed bilayer system without the NPs is shown in Figure 2(b). We see that significant phase separation occurs after $800 \mathrm{~ns}$, we see an increase in the curvature of the bilayer corresponding to a greater phase separation. The analogous simulation with the hydrophobic NPs for $R=1.0,1.5$ and $2.0 \mathrm{~nm}$ are shown in Figures 2(b), (c) and (d) respectively. With the entry of the NP into the bilayer center we see the formation of a cap for the $R=1.0 \mathrm{~nm}$ example (at $500 \mathrm{~ns}$ ), and a full budding/micelle formation in the case of the $R=2.0 \mathrm{~nm}$ example. The $R=1.5 \mathrm{~nm}$ shows the intermediate case, showing the formation of a significant cap around the NP. By measuring the the density of each type of surfactants around the NP, we can measure the dynamic change in the conformation of 
the cap/bud. Figure 3 shows the simulation snapshots at $t=500$ ns for when the NPs are restrained at the center of the bilayer, which clearly indicates the preference of the NP to the domain interface. As with the restrained simulations, we can observe that the preference for the domain interface is apparent in the free trajectory simulations with the NPs. This is shown through the Voronoi tessellation plots of the surfactant domains in the supporting information (Figure S1). ${ }^{38}$ Hence, it is clear that the energetic preference of the NPs is at the phase boundary of $\mathrm{C}_{12} \mathrm{E}_{2} / \mathrm{C}_{12} \mathrm{E}_{2}-\mathrm{M}$, and that the larger NPs induce a larger curvature in its vicinity. To support the assertion that the mixed nature of the bilayer is indeed the cause of the bud/cap formation, we simulated the monocomponent $\mathrm{C}_{12} \mathrm{E}_{2}$ bilayer with each NP (Figure S2) which demonstrates that the budding does not occur.

\section{Membrane Budding and Micelle Formation - The Relation between the Line Tension and the NP}

Figure 4(a) shows the radial distribution function (RDF) profiles of the $\mathrm{C}_{12} \mathrm{E}_{2}$ and $\mathrm{C}_{12} \mathrm{E}_{2}-\mathrm{M}$ components around each NP. For all the NP samples, we see a direction of change in the density profile, where a decrease in the $\mathrm{C}_{12} \mathrm{E}_{2}$ peaks corresponds to a $\mathrm{C}_{12} \mathrm{E}_{2}$ - $\mathrm{M}$ peaks within $2 \mathrm{~nm}$ of the NP surface, which suggests a dynamic change of the surfactant conformations on the NP surface. From distances of $4 \mathrm{~nm}$ to $10 \mathrm{~nm}$, we observe a significant trough forming which is most exaggerated in the case of the $R=2.0 \mathrm{~nm}$, which corresponds to the budding of the NP. Figure 4(b) shows the $\mathrm{E}_{\text {adhesion }}$ computation for each system. From this, we can approximate the $\mathrm{E}_{\text {adhesion }}$ as the sum of the $\mathrm{C}_{12} \mathrm{E}_{2} / \mathrm{C}_{12} \mathrm{E}_{2}-\mathrm{M}$ contributions, which approximates to, on average, values in the ranges of -25 to $-50,-100$ to -140 and -125 to $-175 \mathrm{k}_{B} \mathrm{~T}$ for the $R=1.0,1.5$ and $2.0 \mathrm{~nm}$ examples respectively, for the individual $\mathrm{C}_{12} \mathrm{E}_{2} / \mathrm{C}_{12} \mathrm{E}_{2}-\mathrm{M}$ components. In each case, we see a negative adhesion energy which favours

the wrapping of the NP. ${ }^{39}$ The $\gamma$ measurements were computed from the average of the stabilized phase-boundary bilayer simulations, as seen in Figure 4(c). The estimated value for $\gamma$ for the length of the domain interface is $2.9607 \times 10^{-12} \mathrm{~N}$. Experimental measurements 
of the line tension values range in the region of $0.2-6.2 \times 10^{-12} \mathrm{~N},{ }^{40,41}$ which shows that the mixed bilayer showed in this simulation is comparable to the measurements seen with experiments. The effect of the hydrophobic NPs is such that it introduces a surface that allows the surfactant components to reorganize itself to create a domain interface around it, as shown by the gradual increase of the RDF plots of the $\mathrm{C}_{12} \mathrm{E}_{2} / \mathrm{C}_{12} \mathrm{E}_{2}-\mathrm{M}$ as a function of time (Figure 4(a)). If we break down the influence of the hydrophobic NP upon a mixed bilayer, the locational preference of the NP near the domain interface indicates that it is a line-active component, and hence, reflects a local reduction of the line tension. The resulting composition profile around each NP can be analyzed over time, as seen in Figure 5. The $\phi_{\mathrm{av}}$, measured as a function of distance from the NP center, has been averaged over $1000 \mathrm{~ns}$ in $200 \mathrm{~ns}$ batches. The gradient of $\phi$ seems to play a key part in the budding process. For the $R$ $=1.0 \mathrm{~nm}$, We see the formation of a gradient between $4 \mathrm{~nm}$ and $6 \mathrm{~nm}$ between $600-800 \mathrm{~ns}$, and the formation of such a gradient seems to correspond with the point in the simulation where budding does indeed occur. In the case of the $R=1.5 \mathrm{~nm}$, we see similar effects with a gradient starting at $3 \mathrm{~nm}$, which converges to 0 between $7-8 \mathrm{~nm}$. Finally, for the $R=$ $2.0 \mathrm{~nm}$, we see a clear gradient formed between $4.5-8 \mathrm{~nm}$ between 0 - $400 \mathrm{~ns}$. It is worth noting that the formation of a 'stable' gradient (where the composition gradient persists over the simulation time) is apparent in the case for the $R=1.5 \mathrm{~nm}$ and $2.0 \mathrm{~nm}$, while we see a greater fluctuation in the case of the $R=1.0 \mathrm{~nm}$. This supports our previous analysis in the RDF profiles, which suggests dynamic and continuous rearrangements of the surfactants around the NP radius. To determine that the mismatch between the $\mathrm{C}_{12} \mathrm{E}_{2}$ and $\mathrm{C}_{12} \mathrm{E}_{2}-\mathrm{M}$ was the factor responsible for the budding phenomena, a set of additional simulations were run switching the $\mathrm{C}_{12} \mathrm{E}_{2}-\mathrm{M}$ potentials to $\mathrm{C}_{12} \mathrm{E}_{2}$ with starting configurations at 500 ns of the initial simulations, which show the original trajectories in the process of bud formation, and is shown in the supplementary information (Figure S3). We observe that the budding process is suppressed, especially seen in the case of $\mathrm{R}=2.0 \mathrm{~nm}$ simulations where the vesicle formation stops. 


\section{Order Parameters}

The change in order parameters for each NP simulation can be seen in Figure 6. We observe that the average order parameters are affected approximately $1.5-2 \mathrm{~nm}$ from the surface of the NPs, with a greater change in the order parameter observed with larger NPs. This follows similar patterns of order parameter change seen from studies by Madsen et al, ${ }^{42}$ which analyzed the effect of influenza A M2 transmembrane proteins in a phase separated bilayer, act as an inducer and a sensor for local curvature, and lines up near the sites of line tension/domain interface, to reduce the tensile and compressive stresses in the bilayer due to its conical shape. From the order parameter studies, we can see similar lipid order softening and mixing gradients in the vicinity of the NPs, which indicates that similar effects may be driving the observed NP budding simulations. The key difference between the studies is the shape of the NP and that in their study, the interface was between an $l_{o} / l_{d}$ interface, while here we have simulated a $l_{d} / l_{d}$ interface. Reflecting on this work, we hypothesize that the local reduction of the line tension drives the initial entry of $\mathrm{NP} /$ glycoprotein structures. Further evidence of the importance of energetic mismatch between lipid components within and between each leaflet has been demonstrated by Siggel et al, who demonstrated that a POPC spherical vesicle may dissipate the lipid number asymmetry by the introduction of a lipid scrambling substrate (nhTMEM16 lipid scramblase) which acts to catalyze the number of flip-flop events, which may be seen as the opposite effect to the phenomena induced by the NPs here. ${ }^{43}$

\section{Budding Energetics}

Taking the center of the NP in each simulation as the cap/bud center, we can estimate the curvature $\mathrm{C}$ as $\frac{1}{2.8 \mathrm{~nm}}, \frac{1}{3.3 \mathrm{~nm}}$ and $\frac{1}{3.8 \mathrm{~nm}}$ for the $R=1.0,1.5$ and $2.0 \mathrm{~nm} \mathrm{NPs}$, which is equivalent to $0.357 \mathrm{~nm}^{-1}, 0.303 \mathrm{~nm}^{-1}$ and $0.2 \mathrm{~nm}^{-1}$ respectively - the schematic for these estimates are shown in Figure 5. The computed parameter values from this is shown in Table

2. The membrane budding model suggested by Lipowsky ${ }^{34}$ showed that the competition 


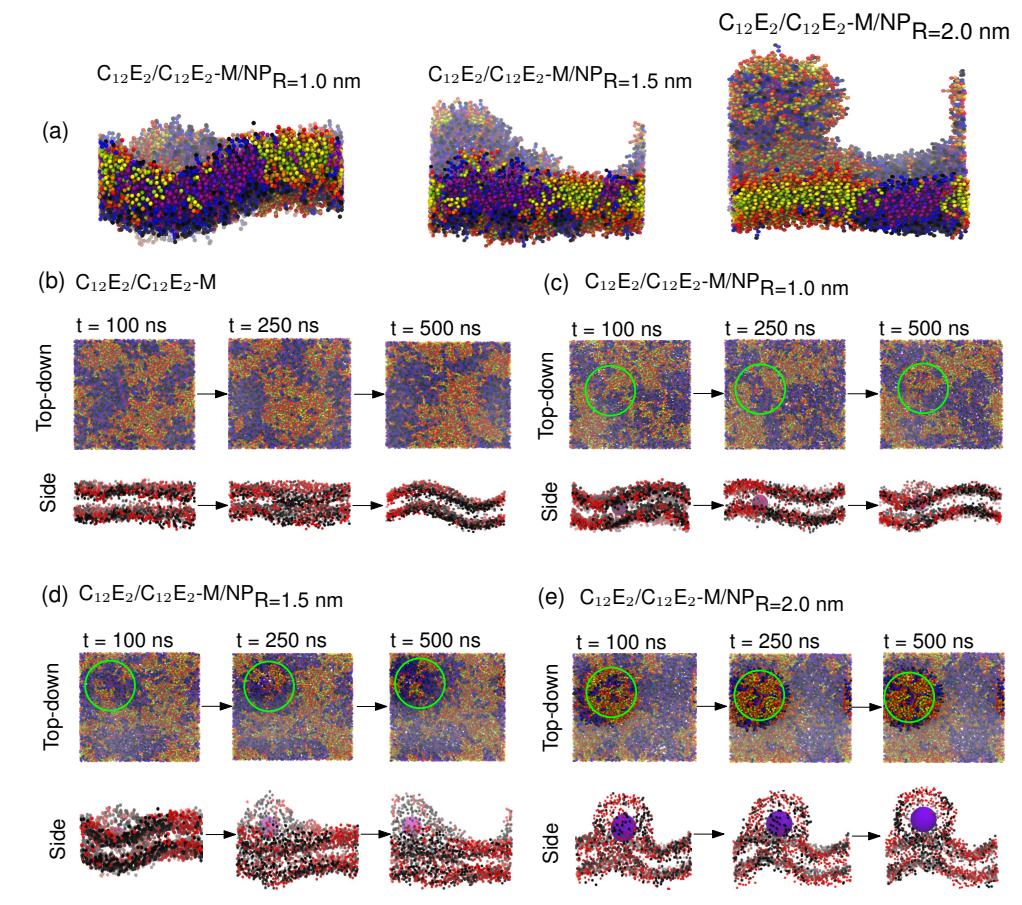

Figure 2: Trajectory snapshots for the series of unrestrained simulations run in this work, over $500 \mathrm{~ns}$, showing the top-down and side views. (a) shows the snapshots of the systems at $500 \mathrm{~ns}$ for the $R=1.0,1.5 \mathrm{~nm}$ and $2.0 \mathrm{~nm}$ simulations, (b) shows the NP-absent simulation, (c) shows the $R=1.0 \mathrm{~nm}$ simulation, (d) shows the $R=1.5 \mathrm{~nm}$ simulation, and (e) shows the $R=2.0 \mathrm{~nm}$ simulations respectively. All simulations were visualized using VMD. ${ }^{44}$
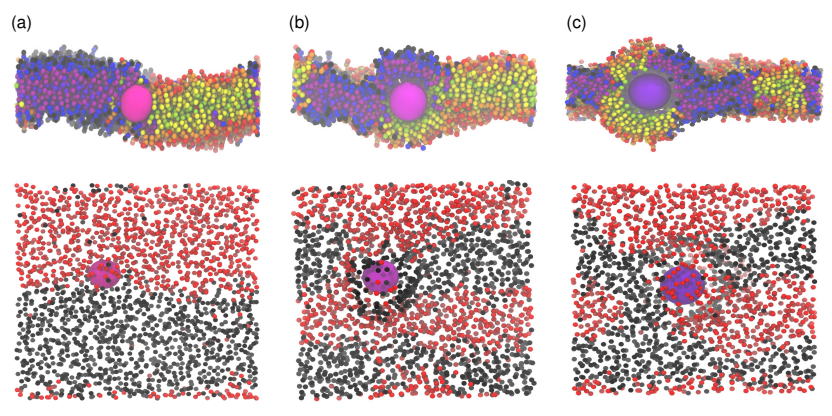

Figure 3: Simulation snapshots at $500 \mathrm{~ns}$ for the $R=1.0,1.5$ and $2.0 \mathrm{~nm} \mathrm{NP}$ simulations shown in (a), (b) and (c) respectively, where the NPs are restrained at the center of the bilayer in the normal plane. 


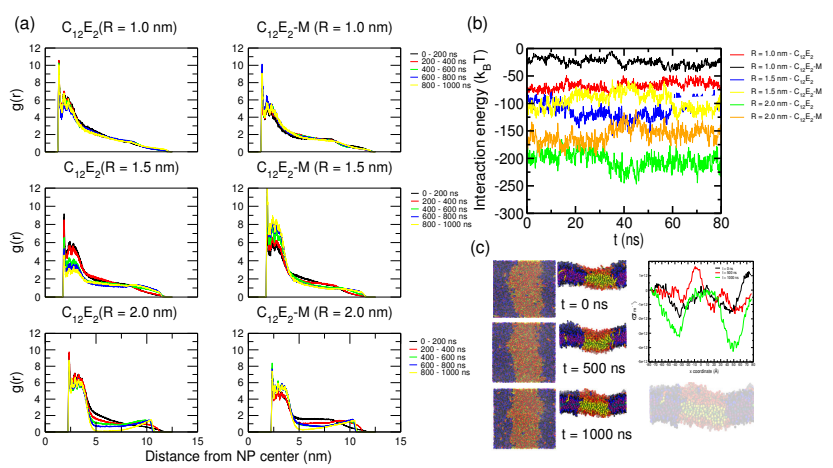

Figure 4: (a) shows the changes in the RDF profiles of the $\mathrm{C}_{12} \mathrm{E}_{2}$ and $\mathrm{C}_{12} \mathrm{E}_{2}-\mathrm{M}$ as a function of distance from the NP center, for the $R=1.0,1.5$ and $2.0 \mathrm{~nm}$ simulations, (b) shows the computed interaction energy between the $\mathrm{C}_{12} \mathrm{E}_{2} / \mathrm{C}_{12} \mathrm{E}_{2}-\mathrm{M}$ residues and the NP over 70 ns, while (c) shows the line tension $(\gamma)$ measurement from a well-separated phase separated system of $\mathrm{C}_{12} \mathrm{E}_{2} / \mathrm{C}_{12} \mathrm{E}_{2}-\mathrm{M}$, averaged at 3 timesteps.
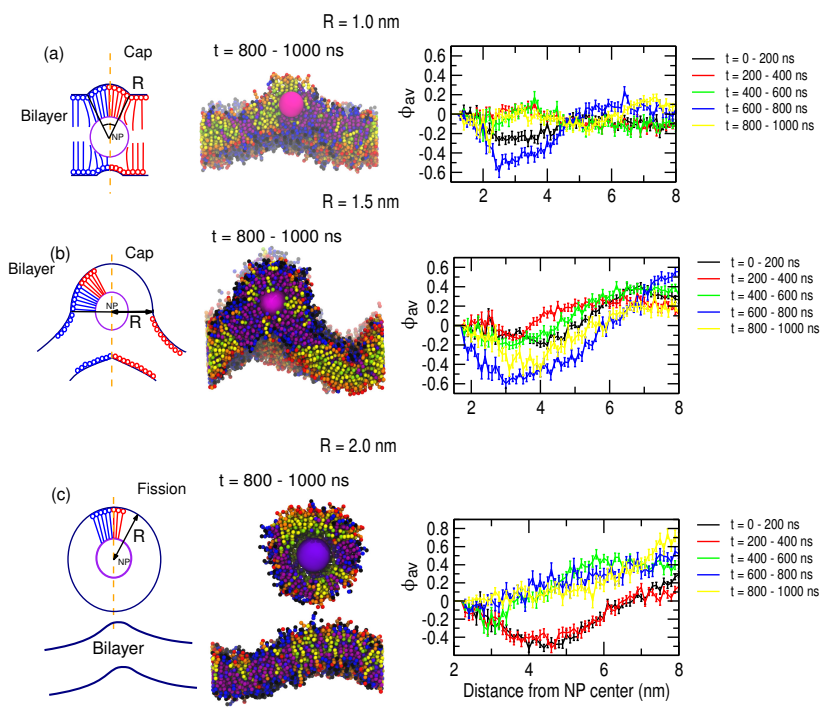

Figure 5: Schematics and snapshots showing the budding formations at between $t=800$ - $1000 \mathrm{~ns}$ for the $R=1.0,1.5$ and $2.0 \mathrm{~nm}$ for (a), (b) and (c) respectively, along with the averaged composition profiles as a function of the distance from the NP center. The value of $R$ represents the radius of the cap/bud used for the curvature computations. The $\phi$ values were averaged over steps of $200 \mathrm{~ns}$ increments. The red components shows the $\mathrm{C}_{12} \mathrm{E}_{2}$ surfactants, while the blue components show $\mathrm{C}_{12} \mathrm{E}_{2}-\mathrm{M}$ components. 

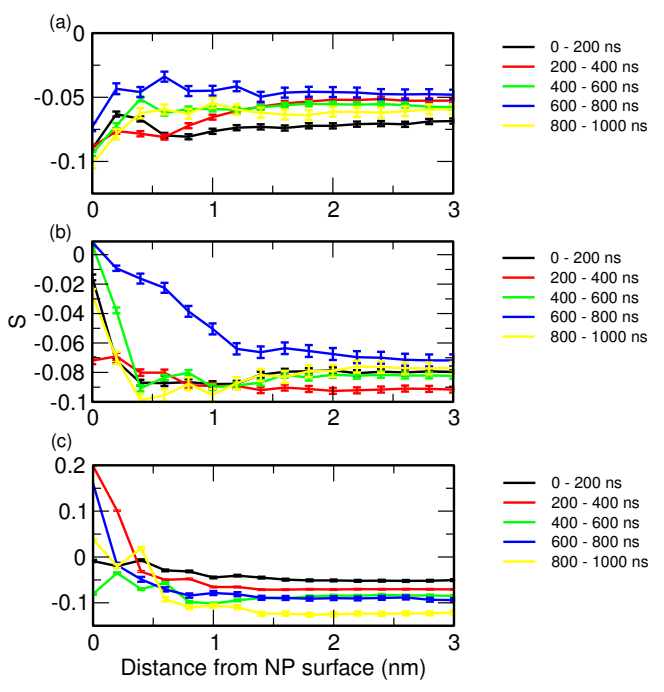

Figure 6: Averaged order parameters of the surfactant chains averaged over $200 \mathrm{~ns}$ bins as a function of distance from the NP surface for the $R=1.0,1.5,2.0 \mathrm{~nm}$ NPs, shown in (a), (b) and (c) respectively

between the domain line tension and the membrane bending energy results to a transition to either a partial or complete budding state. It has been hypothesized that once the domain region exceed a critical size, the domain can transition into a dimpled or fully budded state, or in other words, become unstable and hence undergo a invagination process driven primarily by the fluctuation in the bilayer curvature by changes in temperature. In our simulations, we observe dimpled states of differing magnitudes of curvature with the $R=$ 1.0 and $1.5 \mathrm{~nm} \mathrm{NP}$, and a fully budded state with the $R=2.0 \mathrm{~nm} \mathrm{NP}$ example. As the NPs becomes larger, the budding becomes more significant. Here, the contributing forces in the cap/bud formation are the unfavourable interactions between the $\mathrm{C}_{12} \mathrm{E}_{2} / \mathrm{C}_{12} \mathrm{E}_{2}-\mathrm{M}$, the energetic cost of forming the cap/bud, the effect of the NP upon the local arrangement of the surfactants around it, and the extent of interaction between the NP surfaces and the $\mathrm{C}_{12} \mathrm{E}_{2} / \mathrm{C}_{12} \mathrm{E}_{2}-\mathrm{M}$ components. Figures $7(\mathrm{a})$, (b) and (c) shows the estimated $\mathrm{E}_{\text {total }}$ for a range of spontaneous curvature (between 0 and 0.2 ) and variable $\phi$ values, for the $R=1.0,1.5$ and $2.0 \mathrm{~nm}$ NPs present. We see that the energies range between $78.81-100.6 \mathrm{k}_{b} \mathrm{~T}, 80.8$ - $110.1 \mathrm{k}_{b} \mathrm{~T}$, and $55.08-95.2 \mathrm{k}_{b} \mathrm{~T}$ for the $\mathrm{R}=1,1.5$ and $2.0 \mathrm{~nm}$ simulations respectively. The energetic scale for the simulations show an estimate of $4.1814 \times 10^{-21} \mathrm{k}_{b} \mathrm{~T}$, which would 
suggest that budding would not occur simply through thermal fluctuations overcoming the $\mathrm{E}_{\text {total }}$, as we see with our examples with the NP-absent simulations. Hence, the NPs and how it influences the structure and arrangement of the surfactants near the interface can be identified as the clear catalyst for the budding events. The direct experimental evidence
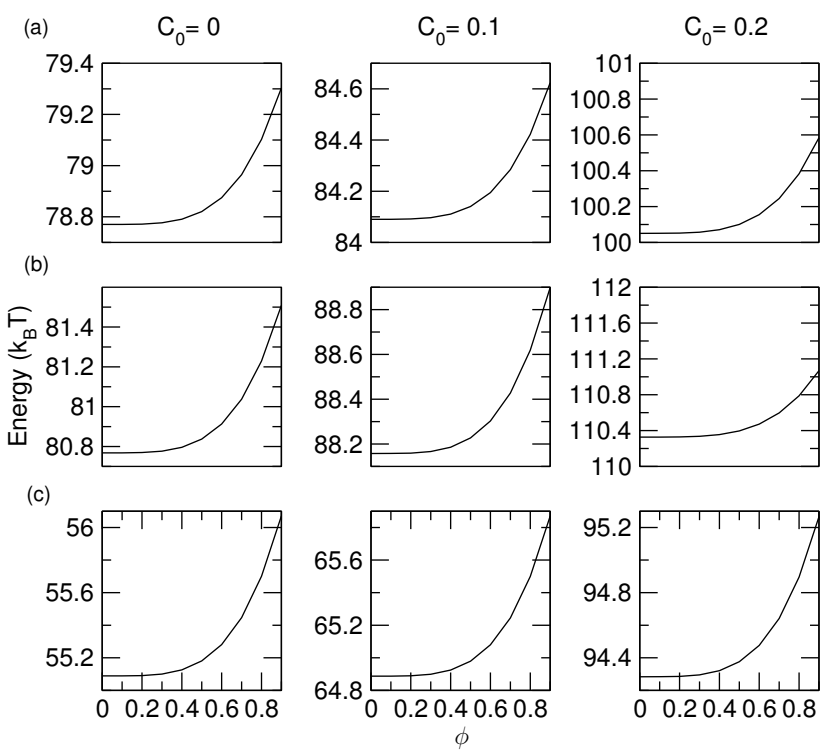

Figure 7: The plots for $\mathrm{E}_{\text {total }}$ for differing spontaneous curvatures $\left(\mathrm{C}_{0}\right)$ and mixtures $(\phi)$ for the $\mathrm{R}=1.0,1.5,2.0 \mathrm{~nm}$ shown in (a), (b) and (c) respectively.

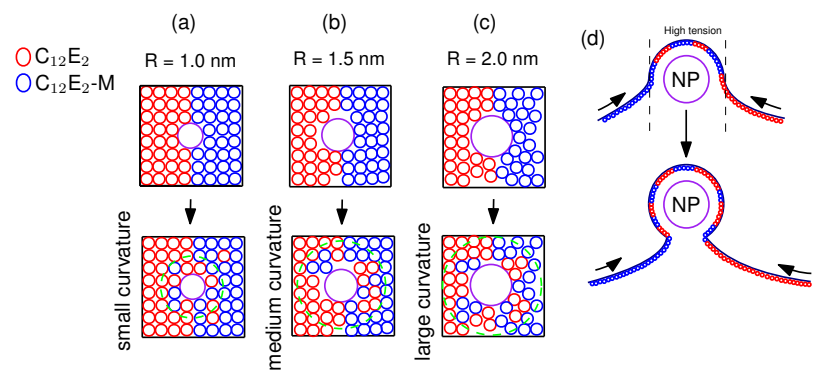

Figure 8: The schematics for the composition of $\mathrm{C}_{12} \mathrm{E}_{2}$ and $\mathrm{C}_{12} \mathrm{E}_{2}-\mathrm{M}$ around each NP through the budding process. (a), (b) and (c) shows the composition arrangement for the $R$ $=1.0,1.5$ and $2.0 \mathrm{~nm}$ respectively. The green circles indicate regions where $\phi_{1} \neq \phi_{2}$, and therefore has the highest spontaneous curvature. (d) shows the budding process with the $R$ $=2.0 \mathrm{~nm} \mathrm{NP}$, where the local stress induced by the NP due to composition mismatch and increase of unfavourable contacts between the surfactants drive the fission process.

of interface driven budding is sparse, due to the difficulty of capturing the phenomena at this scale. However, inferential evidence for the aggregation of NPs at the domain interface 
and the preference for sites of curvature has been demonstrated in previous studies. For example, Palmieri et $a l^{45}$ showed through ternary composition bilayer simulations that lineactive components are observed to accumulate on the binary interface, and following the language of Hassan-Zadeh et $a l,{ }^{14}$ we can conclude that the hydrophobic NP acts as a 'loosening' type of lineactant, compared to hybrid lipids which may act to decrease the line tension by partitioning inside the domain structure. Further evidence of the general nature of the interface nucleating effect of hydrophobic NPs has been observed by Cheung ${ }^{16}$ using dissipative particle dynamics (DPD) simulations, which demonstrated that the nucleation is initially driven the hydrophobic attraction between the NP and each of the lipid/lipid mimic components, and in large numbers, clustering of NPs along the interface has been shown to affect the size of the patches formed in the simulations.

The hydrophobic interface provided by the NP here can be compared to the binding motifs on proteins such as the HIV glycoprotein (HIV-GP41), which binds specifically to CHOL molecules, ${ }^{46}$ whilst other proteins have been known to bind to sphingolipids and sphingomyelin components. ${ }^{47,48}$ In other work, Jeffrys et al suggested that in the presence of the membrane-associated G-protein NRas, the rate of domain formation/phase separation in a tertiary bilayer was shown to be dampened, ${ }^{12}$ which is consistent with its hypothetical effect as a line active component in a mixed bilayer. Hence, we see a consistent effect of preferential positioning near the $l_{o} / l_{d}$ interfaces for both hydrophobic NPs and hydrophobic membrane proteins. Of particular interest, we observe a similar mechanism of budding seen in the study by Madsen et al, ${ }^{42}$ who observed that M2 influenza A proteins preferentially locates to sites of membrane curvature, which are also sites for the co-localization of soft lipids. This is consistent with our observation of a mixing gradient forming around the NP. This study also observed a band of lower tail order region in the direct proximity to the M2 proteins, which would indicate that similar packing defects are present around the NPs shown here, which would drive a entropic sorting mechanism near the vicinity of the NPs. A similar 'wedging' mechanism is therefore hypothesized to be in effect with the larger NPs. The further scission 
and full budding for the NPs in contrast to the M2 influenza protein may be explained by the fact that the $\mathrm{C}_{12} \mathrm{E}_{2}$ and $\mathrm{C}_{12} \mathrm{E}_{2}-\mathrm{M}$ surfactants have identical tail stiffnesses, which has the effect of causing dynamical buckling in the upper hemisphere of the NPs, shown by the changes in RDF profiles and $\mathrm{E}_{\text {interaction }}$ shown in Figures 4(a) and (b) respectively, which we hypothesize have the effect of increasing the neck tension of the dimple/bud. The curvature sensitive nature of proteins has also been seen in NPs, which indicates that this property may be general for all hydrophobic components embedded within the bilayer. Recently, Lavagna et $a l^{49}$ analyzed the effect of increasing the size of hydrophobic NPs within POPC bilayers it was shown that intermediate and large hydrophobic NPs (larger than $2.88 \mathrm{~nm}$ in diameter, comparable to the larger continuum models studied here) were highly sensitive to regions of high curvature in a membrane, and showed linear or 3D aggregation depending on its size.

It is important to point out the limitations of the models used for this study. One of the key limitations of this study is that the mixed bilayer does not represent a single type of bilayer. For example, experimental studies using neutron scatter measurements detected distinctly separate bending moduli in lateral heterogenities. ${ }^{50}$ Also, for certain mixed compositions, we observe drastically varying bending moduli in mixed bilayers, ${ }^{51}$ ranging from 30 ( $l_{d}$ phases) to $100 \mathrm{k}_{B} \mathrm{~T}$ (lo phases) - of an order of magnitude larger than the bending rigidity observed for this study $\left(5.4 \mathrm{k}_{B} \mathrm{~T}\right) .{ }^{37}$ Other studies indicates complementary, yet differing effects of lineactants. For example, Li et al ${ }^{52}$ showed that hybrid lipid-like lineactant molecules aggregates near the domain boundaries of vesicles, indicating a clear reduction in the local line tension, acts to reduce the curvature near the domain boundary. However, this study does follow previous observations in showing that the hydrophobic NP acts as a lineactant in the presence of local domain-like structures within heterogeneous lipid bilayers, and it could be considered an limiting case of $\mathrm{NP} /$ proteins interacting with domain interfaces. From this theoretical study, it is clear that with a spherical NP, the interplay between the bending energy associated with the membrane curvature around the NP, and the adhesion energy due to favourable hydrophobic interaction is disrupted when placed in a 
multicomponent bilayer. We hypothesize that full budding only occurs with the $R=2.0 \mathrm{~nm}$ as adhesion energy between the NP and the surfactants are not sufficient in the cases of the $R$ $=1.0$ and $R=1.5 \mathrm{~nm}$ NPs. This behaviour may differ in the case of multiple NP scenarios, where curvature sensing properties of hydrophobic NPs may allow additional aggregation of smaller NPs ${ }^{49,53}$ which in turn may induce further budding/invagination events.

\section{Conclusion}

We have used CG-MD simulations to compute the interactions of a continuum model of hydrophobic Hamaker-model NPs with a mixed surfactant bilayer and have noted a novel surfactant budding mechanism, with increasing NP radius. Using the model systems we have managed to replicate membrane protein behaviour within heterogeneous bilayers, where the hydrophobic NPs preferentially locate itself at the domain interface, which would suggest that the phenomena seen in this study is general with hydrophobic materials within a mixed bilayer. The hydrophobic NP induces a gradient of 'mismatch' as the NP radius increases, which materializes as significant composition difference between the monolayers. As seen from estimating the bending and edge energies, these energies do not differ significantly between each NP size, which suggests that the adsorption energy on the NP surface and the increase in the mixing gradient and decrease in the order parameters around the NP is the driver for an entropically driven mechanism for the cap/budding of the NP. In addition, we propose that the observed budding is also enthalpically driven by the rearrangement of the composition of the surfactants around the NP, to reduce the length of the domain interface. ${ }^{34}$ This mechanism demonstrates that phase boundaries in heterogeneous bilayers may act as a marker for hydrophobic species to conglomerate and as a catalyst for budding events, and requires further investigation. 


\section{Conflicts of interest}

There are no conflicts of interest to declare.

\section{Acknowledgements}

The authors acknowledge the Scientific Computing Research Technology Platform at the University of Warwick for providing the computational resources to carry out this work. RN thanks the Royal Society for a University Research Fellowship.

\section{References}

(1) Baumgart, T.; Hess, S. T.; Webb, W. W. Imaging coexisting fluid domains in biomembrane models coupling curvature and line tension. Nature 2003, 425, 821-824.

(2) Zhou, W.; Burke, P. J. Versatile Bottom-Up Synthesis of Tethered Bilayer Lipid Membranes on Nanoelectric Biosensor Devices. ACS Applied Materials 6 Interfaces 2017, 9, 14618-14632.

(3) Dimov, N.; Kastner, E.; Hussain, M.; Perrie, Y.; Szita, N. Formation and purification of tailored liposomes for drug delivery using a module-based micro continuous-flow system. Nature Scientific Reports 2017, 7.

(4) Inci, F.; Celik, U.; Turken, B.; Ozer, H.; Kok, F. Construction of P-glycoprotein incorporated tethered lipid bilayer membranes. Biochemistry and Biophysics Reports 2015, 2, $115-122$.

(5) Mazur, F.; Bally, M.; Stadler, B.; Chandrawati, R. Liposomes and lipid bilayers in biosensors. Advances in Colloid and Interface Science 2017, 2. 
(6) Petaccia, M.; Bombelli, C.; Sterbini, F. P.; Papi, M.; Giansanti, L.; Bugli, F.; Sanguinetti, M.; Mancini, G. Liposome-based sensor for the detection of bacteria. Sensors and Actuators B: Chemical 2017, 248, 247-256.

(7) Simons, K.; Ikonen, E. Functional rafts in cell membranes. Nature 1997, 387, 569 572.

(8) Meer, G. V.; Voelker, D. R.; Feigenson, G. W. Membrane lipids: where they are and how they behave. Nature Reviews 2008, 9, 112 - 124 .

(9) Schmid, F. Physical mechanisms of micro- and nanodomain formation in multicomponent lipid membranes. Biochimica et Biophysica Acta 2016, 509 - 528 .

(10) Hakobyan, D.; Heuer, A. Key Molecular Requirements for Raft Formation in Lipid/Cholesterol Membranes. Plos One 2014, 9, e87369.

(11) Marrink, S. J.; Risselada, H. J.; Yefimov, S.; Tieleman, P. D.; de Vries, A. H. The MARTINI Force Field: Coarse Grained Model for Biomolecular Simulations. Journal of Physical Chemistry B 2007, 111, 7812-7824.

(12) Jefferys, E.; Sansom, M. S. P.; Fowler, P. W. NRas slows the rate at which a model lipid bilayer phase separates. Faraday Discussions 2014, 169, 209-223.

(13) Rosetti, C. M.; Montich, G. G.; Pastorino, C. Molecular Insight into the Line Tension of Bilayer Membranes Containing Hybrid Polyunsaturated Lipids. Journal of Physical Chemistry B 2017, 121, 1587 - 1600.

(14) Hassan-Zadeh, E.; Baykal-Caglar, E.; Alwarawrah, M.; Huang, J. Complex Roles of Hybrid Lipids in the Composition, Order and Size of Lipid Membrane Domains. Langmuir 2014, 30, $1361-1369$.

(15) Barnoud, J.; Rossi, G.; Marrink, S. J.; Monticelli, L. Hydrophobic Compounds Reshape Membrane Domains. PLOS Computational Biology 2014, 10, e1003873. 
(16) Cheung, D. Aggregation of nanoparticles on one and two-component bilayer membranes. Journal of Chemical Physics 2015, 141, 194908.

(17) Bandyopadhyay, S.; Tarek, M.; Lynch, M. L.; Klein, M. L. Molecular Dynamics Study of the Poly(oxyethylene) Surfactant C12E and Water. Langmuir 2000, 16, 942 - 946.

(18) Shinoda, W.; DeVane, R.; Klein, M. L. Coarse-grained molecular modeling of non-ionic surfactant self-assembly. Soft Matter 2008, 4, 2454-2462.

(19) Shinoda, W.; DeVane, R.; Klein, M. L. Multi-property fitting and parameterization of a coarse grained model for aqueous surfactants. Molecular Simulation 2007, 33, 27 36.

(20) Seo, S.; Shinoda, W. SPICA Force Field for Lipid Membranes: Domain Formation Induced by Cholesterol. Journal of Chemical Theory and Computation 2019, 15, 762 $-774$

(21) Alemani, D.; Collu, F.; Cascalla, M.; Peraro, M. D. A Nonradial Coarse-Grained Potential for Proteins Produces Naturally Stable Secondary Structure Elements. Journal of Chemical Theory and Computation 2010, 6, 315-324.

(22) Devane, R.; Klein, M. L.; Chiu, C.; Nielson, S. O.; Shinoda, W.; Moore, P. B. CoarseGrained Potential Models for Phenyl-Based Molecules: I. Parametization using Experimental Data. Journal of Physical Chemistry B 2002, 296, 6386 - 6393.

(23) LeBard, D. N.; Levine, B. G.; Mertmann, P.; Barr, S. A.; Jusufi, A.; Sanders, S.; Klein, M. L.; Panagiotopoulos, A. Z. Self-assembly of coarse-grained ionic surfactants accelerated by graphics processing units. Soft Matter 2012, 8, 2385 - 2397.

(24) Devane, R.; Shinoda, W.; Moore, P. B.; Klein, M. L. Transferable Coarse Grain Nonbonded Interaction Model for Amino Acids. Journal of Chemical Theory and Computation 2009, 5, $2115-2124$. 
(25) Shinoda, W.; DeVane, R.; Klein, M. L. Coarse-grained force field for ionic surfactants. Soft Matter 2011, 7, 6178 - 6186.

(26) Chi-Cheng, C.; Moore, P. B.; Shinoda, W.; Nielson, S. O. Size-dependent hydrophobic to hydrophilic transition for nanoparticles: A molecular dynamics study. Journal of Chemical Physics 2009, 131, 244706.

(27) Hamaker, H. C. The London-van der Waals attraction between spherical particles. Physica 1937, 4, $1058-1072$.

(28) Plimpton, S. Fast Parallel Algorithms for Short-Range Molecular Dynamics. Journal of Computational Physics 1995, 117, 1-19.

(29) Nose, S. A unified formulation of the constant temperature molecular dynamics methods. Journal of Chemical Physics 1984, 81, 511.

(30) Hoover, W. G.; Holian, B. L. Kinetic moments method for the canonical ensemble distribution. Physics Letters A 1996, 211, 253.

(31) Tuckermann, M.; Berne, B. J.; Martyna, G. J. Reversible multiple time scale molecular dynamics. Journal of Chemical Physics 1992, 97, 1990 - 2001.

(32) Wolff, J.; Komura, S.; Andelman, D. Budding of domains in mixed bilayer domains. Physical Review E 2015, 91, 012708.

(33) Wolff, J.; Komura, S.; Andelman, D. Budding transition of asymmetric two-component lipid domains. Physical Review E 2016, 94, 032406.

(34) Lipowsky, R. Budding of membranes induced by intramembrane domains. Journal de Physique II 1992, 2, 1825 - 1840.

(35) Lipowsky, R. Domain-induced budding of fluid membranes. Biophysical Journal 1993, $64,1133-1138$. 
(36) Helfrich, W. Elastic Properties of Lipid Bilayers: Theory and Possible Experiments. Zeitschrift fur Naturforschung C 1973, 208, 214-224.

(37) Kurtisovski, E.; Taulier, N.; Ober, R.; Waks, M.; Urbach, W. Molecular Origin of Model Membrane Bending Rigidity. Physical Review Letters 2007, 98, 258103.

(38) Lukat, G.; Kruger, J.; Sommer, B. APL@Voro: a Voronoi-based membrane analysis tool for GROMACS trajectories. Journal of Chemical Information and Modeling 2013, $11,2908-2925$.

(39) Bahrami, A. H.; Raatz, M.; Agudo-Canalejo, J.; Michel, R.; Curtis, E. M.; Hall, C. K.; Gradielski, M.; Lipowsky, R.; Weikl, T. R. Wrapping of nanoparticles by membranes. Advances in Colloid and Interface Science 2014, 208, 214 - 224.

(40) Heinrich, M. C.; Levental, L.; Janmey, P. A.; Baumgart, T. Critical Exponents for Line Tension and Dipole Density Difference from Lipid Monolayer Domain Boundary Fluctuations. Journal of Physical Chemistry B 2008, 112, 8063 - 8068.

(41) Tian, A.; Johnson, C.; Wang, W.; Baumgart, T. Line tension at fluid membrane domain boundaries measured by micropipette aspiration. Physical Review Letters 2007, 98, 208102.

(42) Madsen, J. J.; Grime, J. M. A.; Rossman, J. S.; Voth, G. A. Entropic forces drive clustering and spatial localization of influenza A M2 during viral budding. Proceedings of the National Academy of Sciences of the United States of America 2018, 115, E8595 - E8603.

(43) Siggel, M.; Bhaskara, R. M.; Hummer, G. Phospholipid Scramblases Remodel the Shape of Asymmetric Membranes. Journal of Physical Chemistry Letters 2019,

(44) Dalke, H. W.; Schulten, K. VMD - Visual Molecular Dynamics. Journal of Molecular Graphics 1996, 14, $33-38$. 
(45) Palmieri, B.; Grant, M.; Afran, S. A. Prediction of the Dependence of the Line Tension on the Composition of Lineactants and the Temperature in Phase Separated Membranes. Physical Review Letters 2014, 30, 11734 - 11745.

(46) Schwarzer, R.; Levental, I.; Gramatica, A.; Scolari, S.; Buschmann, V.; Veit, M.; Herrmann, A. The cholestrol-binding motif of the HIV-1 glycoprotein gp41 regulates lateral sorting and oligomerization. Cellular Microbiology 2014, 16, 1565 - 1581.

(47) Coskun, U.; Gryznek, M.; Dreshsel, D.; Simons, K. Regulation of human EGF receptor by lipids. Proceedings of the National Academy of Sciences of the United States of America 2010, 108, $9044-9048$.

(48) Contreras, F. X.; Ernst, A. M.; Haberkant, P.; Bjorkholm, P.; Landahl, E.; Gonen, B.; Tischer, C.; Elofsson, A.; von Heijne, G.; Thiele, C.; Pepperkok, R.; Wieland, F.; Brugger, B. Molecular recognition of a single sphingolipid species by a protein's transmembrane domain. Nature 2012, 481, $525-529$.

(49) Lavagna, E.; Barnoud, J.; Rossi, G.; Monticelli, L. Size-dependent aggregation of hydrophobic nanoparticles in lipid membranes. Nanoscale 2020, 9452.

(50) Nickels, J. D.; Cheng, X.; Motofian, B.; Stanley, C.; Lindner, B.; Herberle, F. A.; Perticaroli, S.; Feygenson, M.; Egami, T.; Standert, R. F.; Smith, J. C.; Myles, D. A. A.; Ohl, M.; Katsaras, J. Mechanical Properties of Nanoscopic Lipid Domains. ACS Nano 2014, 8, 2161-2175.

(51) G.Khelashvili,; Kollmitzer, B.; Heftberger, P.; Pabst, G.; Harries, D. Calculating the Bending Modulus for Multicomponent Lipid Membranes in Different Thermodynamic Phases. Journal of Chemical Theory and Computation 2007, 91, 258103.

(52) Li, Z.; Gorfe, A. A. Modulation of a Small Two-Domain Lipid Vesicle by Linactants. The Journal of Physical Chemistry B 2014, 118, 9208 - 9036. 
(53) Agudo-Canalejo, J.; Lipowsky, R. Uniform and Janus-like nanoparticles in contact with vesicles: energy landscapes and curvature-induced forces. Soft Matter 2017, 13, 2155 $-2173$. 


\section{Graphical TOC Entry}

Some journals require a graphical entry for the Table of Contents. This should be laid out "print ready" so that the sizing of the text is correct.

Inside the tocentry environment, the font used is Helvetica 8 pt, as required by Journal of the American Chemical Society.

The surrounding frame is $9 \mathrm{~cm}$ by $3.5 \mathrm{~cm}$, which is the maximum permitted for Journal of the American Chemical Society graphical table of content entries. The box will not resize if the content is too big: instead it will overflow the edge of the box.

This box and the associated title will always be printed on a separate page at the end of the document. 